\title{
Anticipating synchronization with machine learning
}

\author{
Huawei Fan $\odot,{ }^{1}$ Ling-Wei Kong $\odot,{ }^{2}$ Ying-Cheng Lai $\odot,{ }^{2}$ and Xingang Wang $\odot^{1, *}$ \\ ${ }^{1}$ School of Physics and Information Technology, Shaanxi Normal University, Xi'an 710062, China \\ ${ }^{2}$ School of Electrical, Computer, and Energy Engineering, Arizona State University, Tempe, Arizona 85287, USA
}

(Received 12 March 2021; accepted 8 June 2021; published 22 June 2021)

\begin{abstract}
In realistic systems of coupled oscillators, it is desired to predict the onset of synchronization where the system equations are unknown, raising the need to develop a prediction framework that is model free and fully data driven. We show that this challenging problem can be addressed with machine learning. In particular, exploiting reservoir computing or echo state networks, we employ a "parameter-aware" scheme to train the neural machine using time series acquired from a small number of distinct asynchronous states in the parameter regime prior to the onset of synchronization. The trained machine can then be used to predict the synchronization transition through tuning the control parameter. We demonstrate the power of the machine learning-based framework using two types of synchronization behaviors: Complete synchronization in coupled identical chaotic oscillators and the phase synchronization in coupled nonidentical phase oscillators, which are representative of the collective dynamics in coupled systems. In addition, we design our numerical experiments such that two transition scenarios are covered: Smooth (second-order) and explosive (first-order) transitions that represent the generic types of phase transition in nonlinear physical systems. A remarkable feature is that, for the network systems exhibiting explosive (first-order) transition, the machine learning scheme is capable of predicting not only the locations of the transition points associated with the forward and backward transition paths but also the hysteresis between the two paths.
\end{abstract}

DOI: 10.1103/PhysRevResearch.3.023237

\section{INTRODUCTION}

As a universal concept in nonlinear and complex dynamical systems, synchronization has attracted a great deal of research and continuous interest over the past decades [1-3]. Synchronization refers to the coherent motion between coupled dynamical units, which emerges normally when the interaction or coupling among the units is sufficiently strong. In the study of synchronization, one of the central tasks is to identify the critical coupling strength where the system is transitioned from desynchronization to synchronization [1-3]. For systems of coupled identical oscillators, the critical coupling for complete synchronization can be analyzed by the method of master stability function [4-6]. For systems of nonlinearly coupled phase oscillators, e.g., the classical Kuramoto model, the critical coupling characterizing the onset of phase synchronization can be determined by the mean-field theory $[7,8]$. Synchronization in complex network systems has also been extensively studied [9], in which the important roles of network structure in synchronization have been revealed. The transition from desynchronization to synchronization can be continuous, e.g., the synchronization error or the system order parameter is changing continuously about the critical

\footnotetext{
*wangxg@snnu.edu.cn

Published by the American Physical Society under the terms of the Creative Commons Attribution 4.0 International license. Further distribution of this work must maintain attribution to the author(s) and the published article's title, journal citation, and DOI.
}

coupling. The transition can also be discontinuous and shows the feature of first-order transition in that, near the the transition point, an infinitesimal variation in the coupling can lead to an abrupt change in the synchronization order parameter. This phenomenon was first observed in systems of globally coupled oscillators $[10,11]$ and was also reported in complex networks [12-14] where the first-order transition occurs when the network links are weighted according to the oscillator's natural frequencies [14]. This phenomenon, known as explosive synchronization [12], is the result of the interplay between network structure and collective dynamics.

In realistic systems, such as the human brain [15], synchronization has important implications to the system functions, and a question of practical interest is whether synchronization can be predicted before its occurrence. Different from theoretical studies in which the equations governing the system dynamics are known a priori, in realistic situations the system equations are often unknown. This raises the challenge to predict synchronization in practical situations where only the time series from a few asynchronous states are available, with the goal of predicting whether the oscillators are synchronizable for a given parameter change. In situations such as this, any prediction attempt must be based on time series data obtained before the system evolves into the synchronous state. We are thus motivated to ask the following question: Given that the system operates in a parameter regime where there is no synchronization, would it be possible to predict, without relying on any model, the onset of synchronization based solely on the time series measured from the asynchronous states? In this paper, we articulate a machine-learning framework based on reservoir computing to provide an affirmative answer to this question. 
The idea and principle of exploiting reservoir computing for predicting the state evolution of chaotic systems were first laid out about two decades ago [16,17]. In recent years, model-free prediction of chaotic systems using reservoir computing has gained considerable momentum [18-31]. It has been shown that compared to the conventional prediction techniques in nonlinear science, reservoir computing has an advantage in both accuracy and efficiency. For instance, the state evolution of chaotic systems can be accurately predicted for about a half dozen or so Lyapunov times by reservoir computing [17], which is much longer than the prediction horizon achieved by the traditional methods of nonlinear time series analysis. An interesting finding in these studies is that, even though reservoir computing is unable to make long-term predictions of the state evolution of a chaotic system, it is still able to replicate the ergodic properties of the system [18], namely, the "climate." This feature makes it possible to reproduce the bifurcation diagram of a nonlinear dynamical system without knowing the equations [32]. Recently, a "parameteraware" scheme was proposed to predict critical transitions and system collapse in nonlinear dynamical systems [33], in which a parameter input channel is incorporated into the standard reservoir computing to "guide" the evolution of the reservoir in the predicting phase. It is shown that the machine trained in the normal functional regime can not only predict the transition point of system collapse but also estimate the average lifetime of the transient. (A similar scheme has also been proposed in Refs. [34,35]). Employing the parameteraware scheme and treating the coupling strength as the control parameter, in the present work we demonstrate that machine learning can be effective at anticipating the transition from desynchronization to synchronization in systems of coupled oscillators. We show that the machine trained on a time series containing a handful of asynchronous states is able to not only predict the critical couplings for synchronization but also reproduce the transition path from desynchronization to synchronization. Considering the model-free nature of the prediction framework and the important implications of synchronization to system functions, the findings may have broad applications.

\section{MACHINE-LEARNING METHOD}

Our reservoir computing machine consists of four modules: The $I / R$ layer (input-to-reservoir), the control module, the hidden layer (the reservoir), and the $R / O$ layer (reservoirto-output). The $I / R$ layer is characterized by $\mathcal{W}_{\text {in }}$, a $D_{r} \times$ $D_{\text {in }}$-dimensional matrix that maps the input vector $\mathbf{u}_{\varepsilon}(t) \in$ $\mathbf{R}^{D_{\text {in }}}$ to the dynamical network in the reservoir hidden layer, where the input vector is acquired from the target system at time $t$ for the specific bifurcation parameter value $\varepsilon$. Different from the conventional schemes where the training data are acquired at a fixed bifurcation parameter [18-31], here we consider the situation of time-dependent bifurcation parameter, i.e., $\varepsilon$ is a step function of time. The elements of $\mathcal{W}_{\text {in }}$ are randomly drawn from a uniform distribution within the range $[-\sigma, \sigma]$. The control module is characterized by the vector $\mathbf{s}=\eta(t) \mathbf{b}$, where $\eta(t)$ is the time-dependent control parameter and $\mathbf{b} \in \mathbb{R}^{D_{r}}$ is a bias vector. In general, the control parameter is related to the bifurcation parameter of the target system by a smooth function, where a convenient choice is simply $\eta(t)=\varepsilon(t)$. Effectively, $\eta(t)$ can be regarded as an additional input component that can be incorporated into the input vector $\mathbf{u}(t)$, where the elements of $\mathbf{b}$ are also drawn randomly from a uniform distribution within the range $[-\sigma, \sigma]$. The network in the hidden layer consists of $D_{r}$ nonlinear elements (nodes), whose dynamics are governed by the rule

$$
\begin{aligned}
\mathbf{r}(t+\Delta t)= & (1-\alpha) \mathbf{r}(t)+\alpha \tanh [\mathcal{A} \mathbf{r}(t) \\
& \left.+\mathcal{W}_{\text {in }} \mathbf{u}_{\varepsilon}(t)+\varepsilon_{k}\left(\varepsilon(t)+\varepsilon_{\text {bias }}\right) \mathbf{b}\right],
\end{aligned}
$$

where $\mathbf{r}(t) \in \mathbb{R}^{D_{r}}$ is the state vector of the network at time $t, \Delta t$ is the time step, $\alpha$ is a leakage parameter, $\varepsilon_{k}$ and $\varepsilon_{\text {bias }}$ are constant parameters defining a linear transformation of $\varepsilon(t)$ before inputting into the reservoir, and $\mathcal{A}$ is a $D_{r} \times D_{r^{-}}$. dimensional matrix characterizing the connecting structure of the hidden layer network. With probability $1-p$, the elements of matrix $\mathcal{A}$ are set to be zero. The symmetric, nonzero elements of $\mathcal{A}$ are drawn from a uniform distribution within the range $[-1,1]$ and are normalized so as to make the spectral radius of the matrix equal $\rho$. The output layer is characterized by a $D_{\text {out }} \times D_{r}$-dimensional matrix $\mathcal{W}_{\text {out }}$, which generates the $D_{\text {out }}$-dimensional output vector $\mathbf{v}(t)$ via the rule

$$
\mathbf{v}(t+\Delta t)=\mathcal{W}_{\text {out }} \cdot \mathbf{f}[\mathbf{r}(t+\Delta t)],
$$

where $\mathbf{f}(\mathbf{r})$ is the output function. Following Ref. [22], here we set $\mathbf{f}(\mathbf{r})=\mathbf{r}$ for the odd nodes in the reservoir and $\mathbf{f}(\mathbf{r})=\mathbf{r}^{2}$ for the even nodes, so as to improve the learning performance. As in previous studies of chaos prediction [18-31], we set $D_{\text {in }}=D_{\text {out }}$, such that in the predicting phase the input vector $\mathbf{u}_{\varepsilon}(t)$ can be replaced by the output vector $\mathbf{v}(t)$ directly. The elements of the output matrix are to be determined through a training process. In particular, different from $\mathcal{W}_{\text {in }}$, the elements of $\mathcal{W}_{\text {out }}$ are not known a priori, but are to be "learned" from the input data through a training process, with the purpose to find the proper matrix $\mathcal{W}_{\text {out }}$ such that the output vector $\mathbf{v}(t+\Delta t)$ as calculated from Eq. (2) is as close as possible to the input vector $\mathbf{u}(t+\Delta t)$ for $t=(\tau+1) \Delta t, \ldots,(L-$ 1) $\Delta t, L \Delta t$, where $T_{0}=\tau \Delta t$ is the initial period discarded to remove the transient behavior in the reservoir's response to the training signal, and $L$ is the length of the training time series. This can be done $[18,19,22]$ by minimizing a cost function with respect to $\mathcal{W}_{\text {out }}$, which gives

$$
\mathcal{W}_{\text {out }}=\mathcal{U} \mathcal{F}^{T}\left(\mathcal{F} \mathcal{F}^{T}+\lambda \mathcal{I}\right)^{-1},
$$

where $\mathcal{F}$ is the $D_{r} \times L$-dimensional state matrix whose $k$ th column is $\mathbf{f}\{\mathbf{r}[(\tau+k) \Delta t]\}, \mathcal{U}$ is the $D_{\text {in }} \times L$-dimensional matrix whose $k$ th column is $\mathbf{u}[(\tau+k) \Delta t], \mathcal{I}$ is the identity matrix, and $\lambda$ is the ridge regression parameter.

After training, the elements in matrix $\mathcal{W}_{\text {out }}$ are fixed, and the machine is ready for prediction. In the prediction phase, we first set the control parameter to the values used in the training phase and check the performance of the trained machine. The machine is regarded as successfully trained if it is able to accurately predict the state evolution of the target system for several Lyapunov times with relative error less than $5 \%$ for all training parameters (see Supplemental Material for details [36]). Provided that the reservoir is successfully trained, we then set the control parameter to a specific value of interest (not the parameter values used in the training phase) 

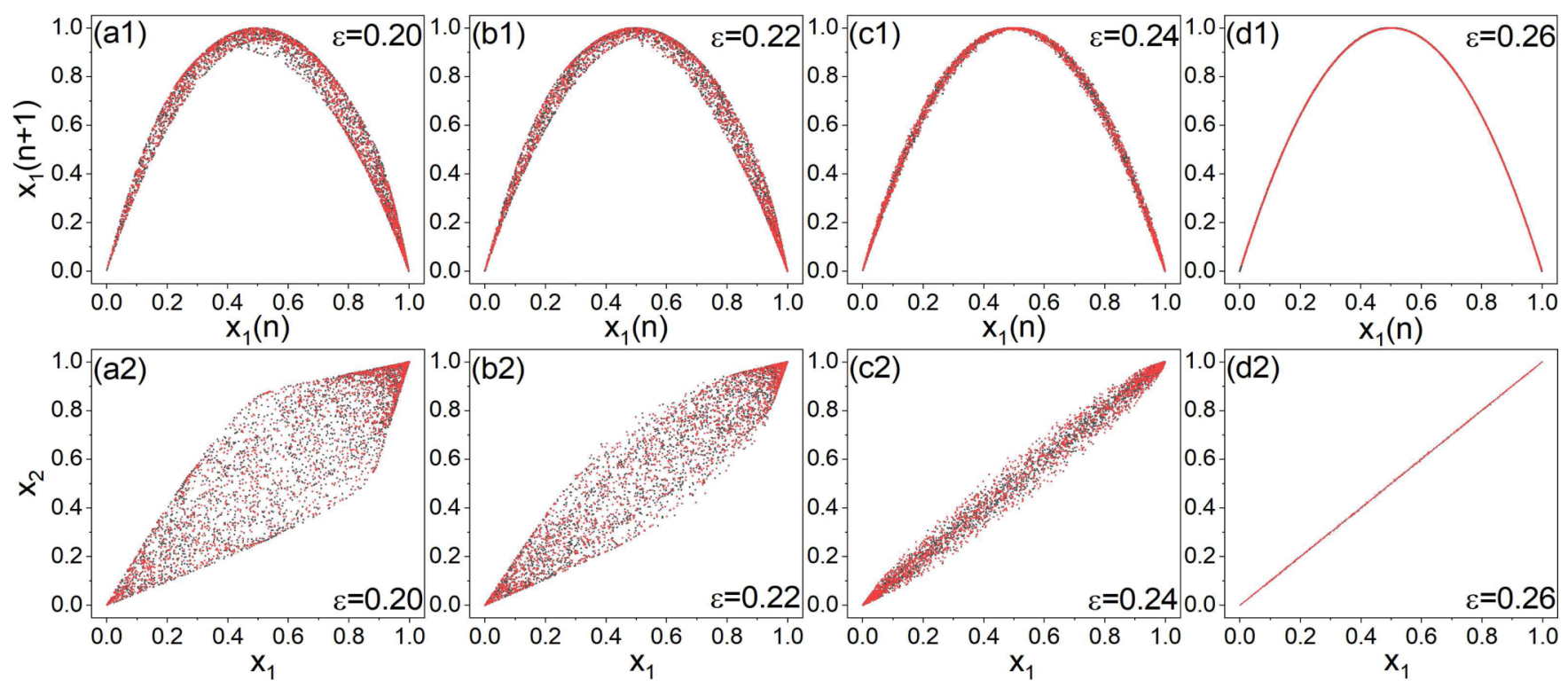

FIG. 1. Predicted synchronization behavior for different values of the bifurcation parameter in coupled identical chaotic maps. The system consists of a pair of coupled chaotic logistic maps with coupling parameter $\varepsilon$ and respective dynamical variables $x_{1}$ and $x_{2}$. Top row (a1-d1): The predicted (black dots) and true (red dots) returned maps constructed from $x_{1}$ for $\varepsilon=0.2,0.22,0.24$, and 0.26 , respectively; bottom row (a2-d2): The predicted (black) and true (red) mutual relationships between $x_{1}$ and $x_{2}$ for the same set of parameter values, where a diagonal line represents complete synchronization.

and then evolve the machine according to Eq. (1) by replacing $\mathbf{u}_{\varepsilon}(t)$ with $\mathbf{v} t$. Finally, by tuning $\varepsilon$ to different values, we monitor the variation of the statistical properties of the reservoir outputs and predict the transition of the system dynamics with respect to the control parameter $\varepsilon$.

The main feature of our reservoir computing is that the input data in the training phase contain two components: (1) The input vector $\mathbf{u}_{\varepsilon}(t)$ representing the time series measured from the target system and (2) the bifurcation parameter $\varepsilon(t)$ marking the condition under which $\mathbf{u}_{\varepsilon}(t)$ is obtained, whereas in the conventional scheme [18-31], only the first component (time series from a fixed value of the bifurcation parameter) is present. In particular, $\mathbf{u}_{\varepsilon}(t)$ consists of $m$ segments of equal length $T$ (i.e., $L=m T$ ) and, for each segment, the value of the bifurcation parameter $\varepsilon(t)$ is fixed so that overall, $\varepsilon(t)$ is a step function of time. (The proposed scheme is equally effective when the segments are not of equal length-see Supplemental Material for details [36]). In the predicting phase, the input vector $\mathbf{u}_{\varepsilon}(t)$ is replaced by $\mathbf{v}(t)$ as in the conventional scheme, but the value of the bifurcation parameter $\varepsilon(t)$ is still needed as an input. Since our goal is to predict synchronization among a number of coupled oscillators, the coupling strength $\varepsilon$ is a natural choice for the bifurcation parameter.

\section{RESULTS}

\section{A. Predicting complete synchronization in coupled chaotic maps}

We consider the following system of two coupled, identical chaotic maps:

$$
\mathbf{x}_{1,2}(n+1)=\mathbf{F}\left[\mathbf{x}_{1,2}(n)\right]+\varepsilon\left\{\mathbf{H}\left[\mathbf{x}_{2,1}(n)\right]-\mathbf{H}\left[\mathbf{x}_{1,2}(n)\right]\right\},
$$

where $\mathbf{x}_{1,2}(n)$ denote the dynamical variables of the system at the $n$th iteration and $\mathbf{F}(\mathbf{x})$ and $\mathbf{H}(\mathbf{x})$ are the map and coupling functions, respectively. As an illustrative example, we choose the one-dimensional chaotic logistic map defined on the unit interval, $F(x)=4 x(1-x)$, and set the coupling function to be $H(x)=F(x)$. The critical coupling value for complete synchronization can be obtained using the master stability function [5,6], which gives that complete synchronization occurs for $0.25 \approx \varepsilon_{1} \leqslant \varepsilon \leqslant \varepsilon_{2} \approx 0.75$.

We obtain the training data from three different values of $\varepsilon: 0.2,0.22$, and 0.24 , all in the desynchronization regime. For each value of $\varepsilon$, we collect the state variables $\{\mathbf{u}(n)\}=$ $\left\{x_{1}(n), x_{2}(n)\right\}$ for $T=2 \times 10^{3}$ successive time steps after disregarding a transient of $10^{3}$ time steps. The time series from the three values of $\varepsilon$ are combined to form a single time series which, together with the step function $\varepsilon(n)$ of the coupling parameter, are fed into the reservoir for training the output matrix $\mathcal{W}_{\text {out }}$. In this application, the hyperparameters of the reservoir are chosen as $\left(D_{r}, p, \sigma, \rho, \alpha, \varepsilon_{k}, \varepsilon_{\text {bias }}\right)=$ $\left(100,0.2,1,10^{-5}, 1,1,0\right)$. The regression parameter for obtaining $\mathcal{W}_{\text {out }}$ is $\lambda=1 \times 10^{-5}$.

To predict the synchronization transition, the trained machine must possess the ability to sense the change in the "synchronization climate" of the target system. Figures 1(a1) 1(d1) show the predicted return maps (black dots) constructed from $x_{1}$ for four values of $\varepsilon: 0.2,0.22,0.24$, and 0.26 , respectively, together with the true return maps (red dots). The corresponding plots of the mutual relation between the two maps are shown in Figs. 1(a2)-1(d2). The first three values of $\varepsilon$ are below $\varepsilon_{1}$, so there is no synchronization, and the last value is in the synchronization regime. The reservoir machine predicts these behaviors correctly. Especially, as the value of $\varepsilon$ is increased from 0.2 to 0.26 , the return map gradually evolves into the map function $F(x)=4 x(1-x)$ and the points $\left(x_{1}, x_{2}\right)$ converge to the diagonal line, which are characteristics of complete synchronization. In fact, statistically the black and red dots cannot be distinguished, indicating 
the superior power of the machine to capture the collective dynamics and "climate" of the target system.

Note that the first three $\varepsilon$ values $(0.2,0.22$, and 0.24$)$ are the ones used in training. Thus it may not be surprising that the reservoir is able to predict correctly the distinct dynamical behaviors of the system at these training parameters, i.e., there is no synchronization. What is remarkable is that the last value $\varepsilon=0.26$ is totally "new" to the machine, as it has never been exposed to data from this parameter value, yet it predicts, still quite correctly, that there is synchronization. This means that training at different coupling parameters in the desynchronization regime has instilled into the machine the ability for it to "sense" the "climate" change in the collective dynamics of the target system.

As the reservoir has been trained to capture the "climate" of the collective dynamics in the coupled chaotic map system, it should be able to predict the synchronization transition. In particular, the expectation is that it would predict correctly the two ending points of the synchronization parameter regime $\left(\varepsilon_{1}, \varepsilon_{2}\right)$ at which a transition to synchronization occurs depending on the direction of parameter variation. To demonstrate the successful prediction of the transition point $\varepsilon_{1}$, we fix the output matrix $\mathcal{W}_{\text {out }}$ and increase the value of $\varepsilon$ systematically from 0.18 to 0.27 at the step size $\Delta \varepsilon=$ $1 \times 10^{-3}$. For each $\varepsilon$ value, we let the machine generate a time series of length $T=1 \times 10^{3}$ and calculate the time-averaged synchronization error $\Delta x=\left\langle\left|x_{1}-x_{2}\right|\right\rangle_{T}$. Figure 2(a) shows the machine-predicted $\Delta x$ versus $\varepsilon$ (red circles), where $\Delta x$ decreases continuously for $\varepsilon \gtrsim 0.2$ and becomes zero for $\varepsilon=$ $\varepsilon_{1} \approx 0.25$. For comparison, the true behavior of $\Delta x$ obtained from direct model simulations is also shown in Fig. 2(a). We see that the predicted results agree well with the ground truth for $\varepsilon \gtrsim 0.2$. At the opposite end of the synchronization regime, the reservoir machine does an equally good job in predicting the critical coupling $\varepsilon_{2} \approx 0.75$ as well as the transition behavior, as shown in Fig. 2(b). In Fig. 2(b), the training data are obtained from the coupling values $\varepsilon=0.76,0.78$, and 0.8 . The results in Fig. 2 are thus evidence that a properly trained reservoir has the power to accurately predict the critical point of transition to synchronization.

Note that, in Figs. 2(a) and 2(b), the good agreement between the prediction and the ground truth holds only for parameter values in the vicinity of the transition point. Away from the transition, e.g., for $\varepsilon<0.2$ in Fig. 2(a), the prediction error increases slightly, which is found to be due to the periodic windows that commonly arise in systems of coupled chaotic maps [37]. In general, a more accurate machine prediction can be obtained as $\varepsilon$ approaches the critical point.

The performance of the reservoir machine in predicting the synchronization transition is affected slightly by the number and locations of the training parameter values. We find that, insofar as training is done with data from at least two distinct parameter values, the synchronization transition can be predicted; training with data from increasingly more values of the bifurcation parameter, regardless of the order, can in general improve the prediction accuracy; the closer the training parameter values to the critical point, the more accurate the prediction. We also find that the machine prediction is robust to additive noise perturbations to the system states or the system bifurcation parameters (see Supplemental Material
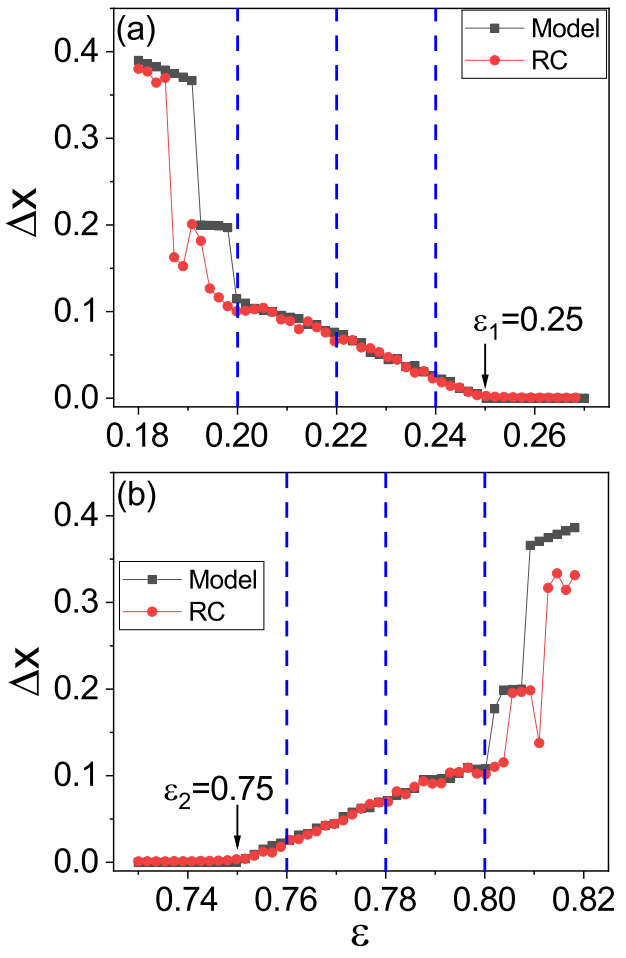

FIG. 2. Predicting synchronization transition in coupled chaotic logistic maps. (a) As the coupling is strengthened, the synchronization error $\Delta x$ gradually decreases and reaches zero at about $\varepsilon_{1} \approx$ 0.25 . (b) The synchronization error $\Delta x$ starts to increase from zero at about $\varepsilon_{2} \approx 0.75$. The vertical dashed lines denote the coupling parameter values used in generating the training data. The machinepredicted results and the ground truth are represented as red circles and black squares, respectively. The machine predicts correctly the transitions at both ends of the synchronization parameter regime $\left(\varepsilon_{1}, \varepsilon_{2}\right)$.

for details about the effects of the number, locations, order of the training parameter values, and noise perturbations on the prediction performance [36]).

\section{B. Predicting synchronization transition in coupled chaotic Lorenz oscillators}

We next consider the synchronization of two coupled identical chaotic Lorenz oscillators. The system dynamics are governed by the equations

$$
\begin{aligned}
& \dot{x}_{1,2}=\mu\left(y_{1,2}-x_{1,2}\right)+\varepsilon\left(x_{2,1}-x_{1,2}\right), \\
& \dot{y}_{1,2}=x_{1,2}\left(\beta-z_{1,2}\right)-y_{1,2}+\varepsilon\left(y_{2,1}-y_{1,2}\right), \\
& \dot{z}_{1,2}=x_{1,2} y_{1,2}-\gamma z_{1,2}+\varepsilon\left(z_{2,1}-z_{1,2}\right),
\end{aligned}
$$

where the parameter setting is $(\mu, \beta, \gamma)=(10,28,2)$ for which an isolated oscillator has a chaotic attractor [38]. Analysis based on the master stability function gives that complete synchronization occurs for $\varepsilon>\varepsilon_{c} \approx 0.42$.

We generate the training data from three distinct values of the coupling parameter in the desynchronization regime: $\varepsilon=0.25,0.3$, and 0.35 . For each parameter value, a time series of length $T=4 \times 10^{3}$ is collected at the time step $\Delta t=0.02$, after disregarding a transient 


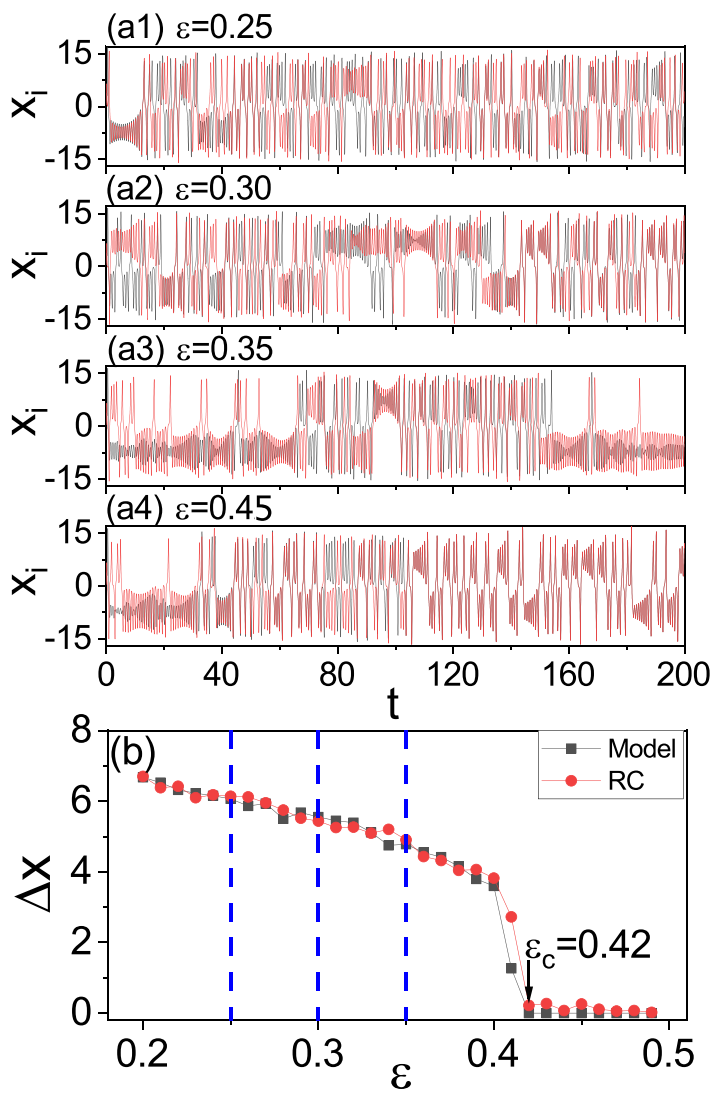

FIG. 3. Predicting synchronization transition in coupled chaotic Lorenz oscillators. (a1-a3) Machine-generated time evolution of $x_{1}$ (black trace) and $x_{2}$ (red trace) for the three training values of the control parameter adopted in the desynchronization regime: $\varepsilon=0.25$, 0.3 , and 0.35 . (a4) Predicted synchronization behavior for $\varepsilon=0.45$. The machine has never been exposed to data from this parameter value, yet it successfully predicts the occurrence of synchronization. (b) Synchronization error $\Delta x$ versus $\varepsilon$. Red circles and black squares represent the machine-predicted and true results, respectively. The three vertical dashed lines indicate the locations of the three training parameters.

phase of length $T_{0}=5 \times 10^{3}$. The input vector $\mathbf{u}(t) \equiv$ $\left[x_{1}(t), y_{1}(t), z_{1}(t), x_{2}(t), y_{2}(t), z_{2}(t)\right]^{T}$ and the control parameter signal $\varepsilon(t)$ are fed into the reservoir machine for training the output matrix $\mathcal{W}_{\text {out }}$. In this example, the hyperparameters of the reservoir are set as $\left(D_{r}, p, \sigma, \rho, \alpha, \varepsilon_{k}, \varepsilon_{\text {bias }}\right)=$ $\left(2 \times 10^{3}, 0.2,0.05,0.1,1,1,0\right)$, and the regression parameter value is set as $\lambda=1 \times 10^{-8}$.

Figures 3(a1)-3(a3) show the predicted evolution of the dynamical variables $x_{1}$ and $x_{2}$ from the two oscillators for the three training parameters. We see that the machine predicts correctly that there is no synchronization for these parameters. When the coupling parameter is set to be $\varepsilon=0.45$ (in the synchronization regime), the machine indeed predicts the synchronous behavior, as shown in Fig. 3(a4). To test if the machine can predict the critical transition point $\varepsilon_{c}$ for synchronization, we increase the control parameter in the machine from 0.2 to 0.5 systematically and calculate the timeaveraged synchronization error $\Delta x=\left\langle\left|x_{1}-x_{2}\right|\right\rangle_{T}$ over a time period of $T=1 \times 10^{4}$ (after discarding a transient period of $\left.T=1 \times 10^{3}\right)$. The dependence of $\Delta x$ on $\varepsilon$ is shown in Fig. 3(b). The machine predicts that the synchronization error approaches zero for $\varepsilon_{c} \approx 0.42$, which is in good agreement with the true value. Similar results are obtained for alternative coupling configurations, e.g., through a single variable or a cross-coupling scheme (see Supplemental Material for details [36]).

\section{Predicting synchronization transition in coupled chaotic food-chain systems}

We next consider the system of two mutually coupled food chains, each with three species [39]:

$$
\begin{aligned}
\dot{x}_{1,2}= & x_{1,2}\left(1-\frac{x_{1,2}}{K}\right)-\frac{a_{c} b_{c} x_{1,2} y_{1,2}}{x_{1,2}+x_{0}}, \\
\dot{y}_{1,2}= & a_{c} y_{1,2}\left[\frac{b_{c} x_{1,2}}{x_{1,2}+x_{0}}-1\right]-\frac{a_{p} b_{p} y_{1,2} z_{1,2}}{y_{1,2}+y_{0}} \\
& +\varepsilon\left(y_{2,1}-y_{1,2}\right), \\
\dot{z}_{1,2}= & a_{p} z_{1,2}\left(\frac{b_{p} y_{1,2}}{y_{1,2}+y_{0}}-1\right)+\varepsilon\left(z_{2,1}-z_{1,2}\right),
\end{aligned}
$$

where $x_{1,2}, y_{1,2}, z_{1,2}$ are the population densities of the resource, consumer, and predator species, respectively. The parameters of the food chain are chosen as [39] $\left(K, a_{c}, b_{c}, a_{p}, b_{p}, x_{0}, y_{0}\right)=(0.99,0.4,2.009$, $0.08,2.876,0.16129,0.5)$, by which the isolated oscillator presents chaotic motion. Using the method of master stability analysis, we have that the critical coupling is $\varepsilon_{c} \approx 8.4 \times 10^{-3}$ and complete synchronization occurs for $\varepsilon>\varepsilon_{c}$.

We generate the training data from three distinct values of the coupling parameter: $\varepsilon=4.5 \times 10^{-3}, 5.5 \times 10^{-3}$, and $6.5 \times 10^{-3}$, all in the desynchronization regime. For each value, we generate a time series of length $T=9600 \Delta t$ with $\Delta t=0.5$ the time step, after disregarding the initial $T_{0}=8000$ steps to remove any transient behavior. All six dynamical variables of the coupled system as well as the coupling strength are fed as input to the reservoir machine. In this example, the hyperparameter values of the reservoir machine are set as $\left(D_{r}, p, \sigma, \rho, \alpha, \varepsilon_{k}, \varepsilon_{\text {bias }}\right)=$ $(1000,0.695,2.30,1.20,0.27,0.049,1.53)$, and the regression parameter is $\lambda=3 \times 10^{-4}$.

We use the trained reservoir to predict the synchronization behaviors in the coupling range $\varepsilon \in[0.004,0.012]$ by calculating the time-averaged synchronization error $\Delta x=$ $\left\langle\left|x_{1}-x_{2}\right|\right\rangle_{T}$ over a time period of $T=2.4 \times 10^{4}$ steps for each parameter value (after discarding transients with $T_{0}=$ $4.8 \times 10^{4}$ steps). Figure 4 shows that the predicted error versus $\varepsilon$ (red circles) is quite close to the true errors (black squares). We see that the synchronization transition point predicted by the machine agrees with the true value.

\section{Predicting explosive synchronization in coupled nonidentical phase oscillators}

The route to complete synchronization treated in Secs. III A, III B, and IIIC belongs to the category of second-order phase transition [1,2], where a physical quantity characterizing the degree of synchronization varies continuously (albeit nonsmoothly) through the transition 


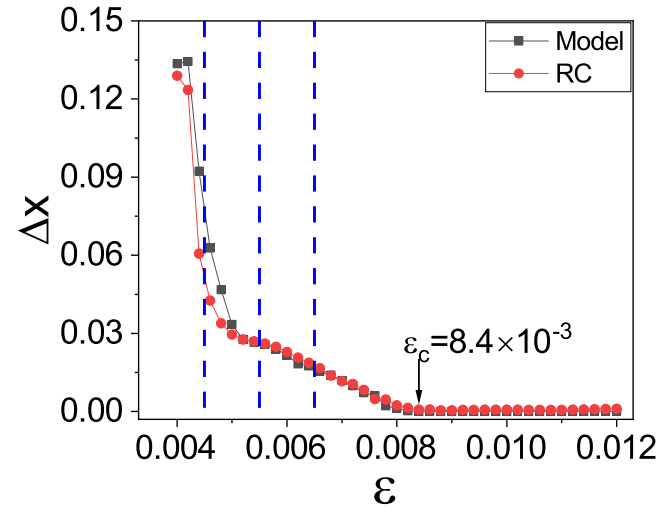

FIG. 4. Predicting synchronization transition in coupled chaotic food chains. Shown are the true synchronization errors (black squares) and reservoir-predicted errors (red circles) for various values of the coupling strength $\varepsilon$. The three vertical blue dashed lines indicate the locations of the training parameters.

point. As demonstrated above, our machine-learning scheme is fully capable of predicting the transition in all the cases (similar results have been obtained for the classical Kuramoto model, where the transition from desynchronization to synchronization is also continuous and the transition behavior can be predicted by the machine based on the time series from several values of the training parameter. Details are given in the Supplemental Material [36]). Another type of synchronization transition that has been extensively studied in the literature is the first-order phase transition, also known as explosive synchronization [12-14], where the onset of synchronization is abrupt in the sense that the underlying characterizing quantity changes discontinuously at the transition point. The question is whether our machine learning scheme can predict explosive synchronization, including the values of the critical couplings and the behavior of the system order parameter around the transition point. Using the paradigmatic system showing explosive synchronization in the literature [12-14], namely, the network of coupled nonidentical phase oscillators, here we present an affirmative answer to this question.

For simplicity, we consider a small star-structure network of $N=4$ nodes, as shown in Fig. 5(a), where the natural frequencies of the peripheral (leaf) nodes are identical and those of the hub node are proportional to its degree [13]. The network dynamics is described by

$$
\begin{aligned}
& \dot{\theta}_{l}=\omega+\varepsilon \sin \left(\theta_{h}-\theta_{l}\right), \\
& \dot{\theta}_{h}=k_{h} \omega+\varepsilon \sum_{l=1}^{3} \sin \left(\theta_{l}-\theta_{h}\right),
\end{aligned}
$$

where $l=1,2,3$ denote the leaf nodes, $h$ denotes the hub node, $\varepsilon$ is the uniform coupling strength, and $k_{h}=3$ is the degree of the hub node. The degree of network synchronization can be characterized by the order parameter

$$
R=\left\langle\left|\frac{1}{N} \sum_{j=1}^{N} e^{i \theta_{j}}\right|\right\rangle_{T},
$$

(a)
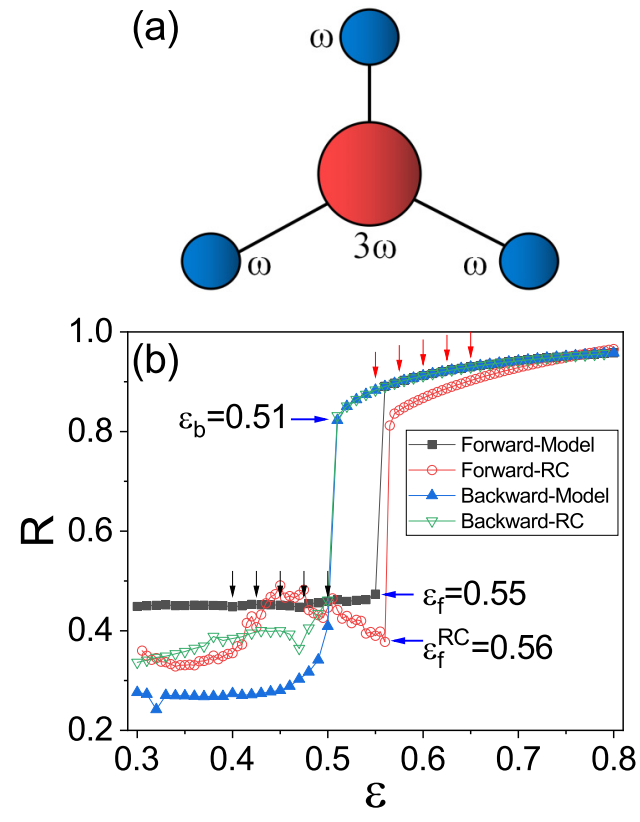

FIG. 5. Predicting explosive synchronization transitions in coupled nonidentical phase oscillators. (a) Star-structure network model. (b) Synchronization order parameter $R$ versus the coupling strength $\varepsilon$ for the forward and backward transition paths. The true transitions obtained from model simulations are represented by black solid squares (forward transition) and blue solid up-triangles (backward transition). The corresponding machine predictions are displayed as red open circles (forward transition) and green empty down-triangles (backward transition). The values of the coupling parameters used to generate the training data for predicting the forward and backward transitions are marked by the black and red arrows, respectively.

where $j=1, \ldots, N$ is the node index, $N=4$ is the network size, $|\cdot|$ is the module function, and $\langle\cdot\rangle_{T}$ denotes the time average.

Setting $\omega=1$, we increase $\varepsilon$ systematically from 0.3 to 0.8 and calculate the dependence of $R$ on $\varepsilon$ by simulating Eq. (9). In numerical simulations, the initial conditions of the oscillators are randomly chosen from the range $(0,2 \pi]$ and the integration time step is $\Delta t=0.05$. Representative numerical results are shown in Fig. 5(b) (black solid squares). It can be seen that, at about $\varepsilon_{f}=0.55$, the value of $R$ changes abruptly from about 0.45 to about 0.9 - the feature of a discontinuous, first-order transition. The dynamical origin of this type of explosive synchronization lies in the interplay between the heterogeneity of the network structure and node dynamics, which occurs naturally in networks where the node degree and the natural frequency are positively correlated [14]. Our goal is to use the reservoir machine trained by the time series from the desynchronization states to predict the critical coupling $\varepsilon_{f}$.

The data used in training consist of time series from five values of $\varepsilon$, all inside the desynchronization regime: $\varepsilon=0.4$, $0.425,0.45,0.475$, and 0.5 . For each $\varepsilon$ value, we collect time series measurements from all nodes: $x_{j}=\sin \left(\theta_{j}\right)$ and $y_{j}=\cos \left(\theta_{j}\right)$, with $j=1, \ldots, 4$. The input state vector therefore is $\mathbf{u}=\left[x_{1}, y_{1}, x_{2}, y_{2}, x_{3}, y_{3}, x_{4}, y_{4}\right]^{T}$. The length of the training data is $1.5 \times 10^{4}$, consisting of time series of five segments, one from each value of $\varepsilon$. The data, together with 
the parameter function $\varepsilon(t)$, are fed into the machine for determining the output matrix $\mathcal{W}_{\text {out }}$. In this example, the parameter values of the reservoir are set as $\left(D_{r}, p, \sigma, \rho, \alpha, \varepsilon_{k}, \varepsilon_{\text {bias }}\right)=$ $(1000,0.2,1,1.15,1,1,0)$, and the regression parameter is $\lambda=1 \times 10^{-10}$.

In the predicting phase, we increase the control parameter $\varepsilon$ systematically from 0.3 to 0.8 with the step $\Delta \varepsilon=$ 0.01 and calculate from the machine output the variation of the synchronization order parameter $R$ with respect to $\varepsilon$. In doing this, we transform the output vector $\mathbf{u}=$ $\left[x_{1}, y_{1}, x_{2}, y_{2}, x_{3}, y_{3}, x_{4}, y_{4}\right]^{T}$ back to the original state variables $\left[\theta_{1}, \theta_{2}, \theta_{3}, \theta_{4}\right]^{T}: \theta_{i}=\arctan \left(y_{i} / x_{i}\right)+\pi / 2$ for $x_{i} \geqslant 0$ and $\theta_{i}=\arctan \left(y_{i} / x_{i}\right)+\pi$ for $x_{i}<0$. The results predicted by the machine are shown in Fig. 5(b) (red open circles). It can be seen that, at about $\varepsilon_{f}^{r c}=0.56$, the value of $R$ changes suddenly from about 0.38 to about 0.8 - the feature of a first-order transition.

A distinct feature of explosive synchronization transition is that when $\varepsilon$ is varied in the opposite direction, the variation of $R$ will follow a different path-the phenomenon of synchronization hysteresis [12-14]. To demonstrate it, we decrease $\varepsilon$ from 0.8 to 0.3 and calculate $R$ by solving Eq. (9) numerically. To observe the hysteresis phenomenon in this context, we apply small random perturbations of amplitude 5\% to the natural frequency of the leaf nodes [13]. Figure 5(b) shows the results (blue solid up-triangles), where the backward and forward transition paths are identical for $\varepsilon>\varepsilon_{f}$, but diverge from each other for $\varepsilon \leqslant \varepsilon_{f}$. Particularly, at $\varepsilon_{f}$, we have $R \approx 0.9$ for the backward path, whereas $R \approx 0.45$ for the forward path. Along the backward path, as $\varepsilon$ decreases from $\varepsilon_{f}$, the value of $R$ maintains at large values until the critical coupling $\varepsilon_{b} \approx 0.51$, where $R$ is suddenly decreased from about 0.8 to about 0.4 . Since $\varepsilon_{b}<\varepsilon_{f}$, a hysteresis loop of width $\Delta \varepsilon \equiv \varepsilon_{f}-\varepsilon_{b}$ thus appears in the parameter region $\varepsilon \in\left(\varepsilon_{b}, \varepsilon_{f}\right)$. The dynamical mechanism for the hysteresis loop is the bistability of the synchronization manifold in this region, deemed as a necessary condition for generating a first-order phase transition [14].

We now test the power of the machine in predicting the backward transition associated with explosive synchronization. In this case, the training data are generated by time series from five coupling parameter values in the strong synchronization regime: $\varepsilon=0.65,0.625,0.6,0.575$, and 0.55 . The input vector is constructed in the same way as for predicting the forward transition, and the hyperparameters of the reservoir are $\left(D_{r}, p, \sigma, \rho, \alpha, \varepsilon_{k}, \varepsilon_{\text {bias }}\right)=$ $(1000,0.2,1,1.15,1,1,0)$ with the regression parameter $\lambda=$ $1 \times 10^{-7}$. We decrease $\varepsilon$ systematically from 0.8 to 0.3 at the step size $\Delta \varepsilon=0.01$. The dependence of $R$ on $\varepsilon$ predicted by the machine is shown in Fig. 5(b) (open green downtriangles). It can be seen that the machine predictions agree well with the true behavior of the backward transition where, at the transition point $\varepsilon_{b}$, the value of $R$ decreases suddenly from about 0.8 to about 0.4 .

\section{DISCUSSION}

We have articulated and tested a model-free, machinelearning scheme to predict synchronization transition in systems of coupled oscillators. The machine is trained with time series collected from a small number of coupling (con- trol) parameter values, all in the desynchronization regime, as well as the value of the control parameter itself through a specially designed input channel. Prediction is achieved by feeding any desired parameter value into the input parameter channel. We have demonstrated that a properly trained machine is able to not only reproduce, statistically, the ergodic properties of the collective dynamics at the training parameter values but also predict, quantitatively, how the collective dynamics change with respect to the variations in the control parameter. Examples demonstrating the predictive power of our machine-learning scheme include complete synchronization in coupled identical chaotic oscillators and explosive synchronization in coupled nonidentical phase oscillators. For complete synchronization, both the critical coupling for synchronization and the variation in the degree of synchronization about the critical point can be well predicted. For explosive synchronization, our scheme not only predicts the forward and backward critical couplings but also reproduces the hysteresis loop associated with a first-order transition. Additional simulations have been carried out to predict phase synchronization in coupled chaotic Rössler oscillators [40], with the result that the machine trained with time series in the asynchronous states is capable of predicting the occurrence of phase synchronization (Supplemental Material [36]).

Reservoir computing-based prediction of chaotic systems is an active field of research at the boundary between nonlinear dynamics and machine learning [18-31]. Distinct from previous studies on predicting the state evolution, the present work focuses on predicting the collective behavior of coupled oscillators. The basic underlying idea is the concept of "climate" replication [18]. The main difference between the current study and Ref. [18] is that the parameter-aware scheme possesses the ability of transfer learning, i.e., the machine trained by the the time series of the asynchronous states is able to predict the "climates" of the synchronous states. This feature is desired in realistic applications, as the knowledge learned from one task ("climate" replication) can be transferred and used to accomplish a different task (synchronization prediction). The current work is also inspired by the studies in Refs. [32-35], where it was shown that the bifurcation behaviors of a dynamical system can be reconstructed based on the time series from a few training parameter values. While our work and Ref. [33] share the same principle, there are some key differences. First, the dynamical phenomena treated in Ref. [33] and in our present work are different, where the former dealt with predicting system collapse (i.e., for a given system parameter, to predict whether the system is in a transient regime and, if yes, how long the average transient lifetime is), but our current study focuses on predicting synchronization transition, i.e., for a given coupling strength to predict whether the oscillators are synchronizable. Prior to our work, it was not apparent if the method in Ref. [33] would actually work for bifurcation scenarios that differ from crisis, especially in coupled systems. Our present work demonstrates that, despite the different bifurcation mechanisms, the parameter-aware machine-learning scheme is also effective for predicting synchronization transition in coupled oscillator systems. This result generalizes Ref. [33] and provides useful insights into the nature of the learning, i.e., it is the dynamics that the machine learns 
from the training data, not the mathematical expressions describing the time series. Second, the types of dynamical systems treated in Ref. [33] and in our present work are different, where the former are "single-component" types of nonlinear dynamical systems, whereas the latter are coupled oscillator systems. As a typical approach to studying the collective dynamics in spatiotemporal systems, network models of coupled oscillators are representative of many real-world systems. Considering the important implications of synchronization to system functions and operations, the finding of the current study may have broad applications. Finally and most importantly, our main results differ from those in Ref. [33]. Besides predicting the location of the transition point as reported in Ref. [33], here we have demonstrated that the characteristics of the transition, e.g., whether it is continuous (second order) or discontinuous (first order), can be predicted. In particular, in the star-network model of coupled phase oscillators, we find that the machine is able to not only predict the critical couplings but also reconstruct the hysteresis loop. Our current study confirms the power of the machine-learning scheme in predicting coupled dynamical systems and highlights the potentials of the parameter-aware scheme in predicting more sophisticated transitions encountered in complex dynamical systems.

We note that there are other model-free approaches in the literature for predicting synchronization transitions. In Ref. [41], a method was proposed for determining the synchronization domain, i.e., the Arnold tongue, of periodically driven oscillators. It was shown that, under certain conditions, the whole Arnold tongue can be reconstructed based on the time series measured from a single asynchronous state. The success of this technique relies on the information and the properties of the driving force. Specifically, the amplitude and frequency of the driving force are required to be known a priori and, to predict the critical coupling, the forcing amplitude should be sufficiently small. In addition, in the implementation of this technique, it is necessary to first estimate the coupling function by quantifying the response of the oscillator to the external driving, which was accomplished via another estimation technique. In our approach of parameter-aware reservoir computing, these restrictions are lifted. Another model-free technique for predicting the onset of synchronization is compressive sensing [42,43], which applies to the general networks of coupled oscillators. Yet this technique applies to networks with sparse connections only and requires that the equations of the nodal dynamics have a simple mathematical structure, i.e., the equations can be represented by a small number of power series or Fourier series terms. These limitations do not exist in our parameter-aware machine-learning scheme.

The type of collective dynamics tested in the present work is complete synchronization between a pair of coupled chaotic oscillators for which the transition is of the nature of second order, and the first order, explosive synchronization in a small network. A possible extension of our work to other types of collective dynamics in large complex systems, such as partial (cluster) synchronization, chimeralike states and spiral waves, is worth pursuing. A difficulty with large systems is the requirement to use large reservoir networks so that the complexity of the machine can "overpower" that of the target system. Quantitatively, how the size of the reservoir network should be enlarged to accommodate an increase in the size of the target system as characterized by, e.g., a scaling law, remains unknown at the present. With the use of large reservoir networks come the issues of data requirement and computation overload, as to train a large reservoir machine not only requires massive data but also imposes a serious demand for the computational resource. One approach to deal with this difficulty is the parallel reservoir computing scheme [22,25]. However, a recent work revealed that the parallel scheme may fail to sense and predict the phase coherence among a pair of coupled, nonidentical chaotic oscillators [30]. It remains a worthy issue to study if the parallel scheme can be exploited to predict the collective dynamics among a large number of coupled oscillators.

It is noteworthy that, for diffusively coupled identical oscillators (e.g., the system of coupled chaotic Lorenz oscillators), the training data must be taken from the desynchronization regime. If the training data are from the synchronization regime, the machine is unable to predict the transition. The reason is that, when the oscillators are completely synchronized, the coupling term becomes zero. As the time series are independent of the coupling parameter, the control parameter loses its function and the reservoir machine is unable to "sense" the change in the dynamical "climate." This is also the case for the model of coupled nonidentical phase oscillators studied in Sec. IIID, in which the coupling term vanishes if the phases of the oscillators are completely synchronized (i.e., $r=1$ ). In Fig. 5, the success in predicting the backward transition relies on the slightly desynchronized nature of the training states (i.e., $r \lesssim 1$ ).

Because of the current lack of a general theoretical (mathematical) understanding of the inner working mechanism of reservoir computing, our confidence in this type of machine learning thus lies entirely in numerical success. In fact, our numerical experiments were designed to be as comprehensive as possible. More specifically, we studied two types of synchronization behaviors: Complete synchronization in coupled identical chaotic oscillators and the phase synchronization in coupled nonidentical phase oscillators, which are representative of the collective dynamics in coupled systems. In addition, we designed our numerical examples such that two transition scenarios are covered: Smooth (second-order) and explosive (first-order) transitions that represent the generic types of phase transitions in nonlinear physical systems. In all the cases studied, a properly trained parameter-aware machine is capable of accurately predicting the synchronization transition. A practical limitation at the present is the system size: If the system is too large, both the amount of required training data as well as computation overload can be quite large.

\section{ACKNOWLEDGMENTS}

This work was supported by the National Natural Science Foundation of China under the Grant No. 11875182. The work at Arizona State University was supported by the Office of Naval Research under Grant No. N00014-21-1-2323. 
[1] Y. Kuramoto, Chemical Oscillations, Waves, and Turbulence (Springer, Berlin, 1984).

[2] A. Pikovsky, M. Rosenblum, and J. Kurths, Synchronization: A Universal Concept in Nonlinear Science (Cambridge University Press, Cambridge, 2003).

[3] S. H. Strogatz, Sync: The Emerging Science of Spontaneous Order (Hyperion, New York, 2003).

[4] L. M. Pecora and T. L. Carroll, Synchronization in Chaotic Systems, Phys. Rev. Lett. 64, 821 (1990).

[5] L. M. Pecora and T. L. Carroll, Master Stability Functions for Synchronized Coupled Systems, Phys. Rev. Lett. 80, 2109 (1998).

[6] L. Huang, Q. Chen, Y.-C. Lai, and L. M. Pecora, Generic behavior of master-stability functions in coupled nonlinear dynamical systems, Phys. Rev. E 80, 036204 (2009).

[7] S. Watanabe and S. H. Strogatz, Integrability of a Globally Coupled Oscillator Array, Phys. Rev. Lett. 70, 2391 (1993).

[8] E. Ott and T. M. Antonsen, Low dimensional behavior of large systems of globally coupled oscillators, Chaos 18, 037113 (2008).

[9] A. Arenas, A. Díaz-Guilera, J. Kurths, Y. Moreno, and C. S. Zhou, Synchronization in complex networks, Phys. Rep. 469, 93 (2008).

[10] H.-A. Tanaka, A.J. Lichtenberg, and S. Oishi, First Order Phase Transition Resulting from Finite Inertia in Coupled Oscillator Systems, Phys. Rev. Lett. 78, 2104 (1997).

[11] D. Pazó, Thermodynamic limit of the first-order phase transition in the Kuramoto model, Phys. Rev. E 72, 046211 (2005).

[12] J. Gómez-Gardeñes, S. Gómez, A. Arenas, and Y. Moreno, Explosive Synchronization Transitions in Scale-Free Networks, Phys. Rev. Lett. 106, 128701 (2011).

[13] Y. Zou, T. Pereira, M. Small, Z. Liu, and J. Kurths, Basin of Attraction Determines Hysteresis in Explosive Synchronization, Phys. Rev. Lett. 112, 114102 (2014).

[14] S. Boccaletti, J. A. Almendral, S. Guan, I. Leyva, Z. Liu, I. Sendiña-Nadal, Z. Wang, and Y. Zou, Explosive transitions in complex networks' structure and dynamics: Percolation and synchronization, Phys. Rep. 660, 1 (2016).

[15] E. R. Kandel, J. H. Schwartz, and T. M. Jessell, Principle of Neural Science, 3rd ed. (Appleton and Lange, Norwalk CT, 1991).

[16] H. Jaeger, The "echo state" approach to analysing and training recurrent neural networks-with an erratum note, Bonn, Germany: German National Research Center for Information Technology GMD Technical Report 148, 13 (2001).

[17] H. Jaeger and H. Haas, Harnessing nonlinearity: Predicting chaotic systems and saving energy in wireless communication, Science 304, 78 (2004)

[18] J. Pathak, Z. Lu, B. R. Hunt, M. Girvan, and E. Ott, Using machine learning to replicate chaotic attractors and calculate lyapunov exponents from data, Chaos 27, 121102 (2017).

[19] Z. Lu, J. Pathak, B. Hunt, M. Girvan, R. Brockett, and E. Ott, Reservoir observers: Model-free inference of unmeasured variables in chaotic systems, Chaos 27, 041102 (2017).
[20] Z. Lu, B. R. Hunt, and E. Ott, Attractor reconstruction by machine learning, Chaos 28, 061104 (2018).

[21] J. Pathak, A. Wilner, R. Fussell, S. Chandra, B. Hunt, M. Girvan, Z. Lu, and E. Ott, Hybrid forecasting of chaotic processes: Using machine learning in conjunction with a knowledge-based model, Chaos 28, 041101 (2018).

[22] J. Pathak, B. Hunt, M. Girvan, Z. Lu, and E. Ott, Model-Free Prediction Of Large Spatiotemporally Chaotic Systems from Data: A Reservoir Computing Approach, Phys. Rev. Lett. 120, 024102 (2018).

[23] T. L. Carroll, Using reservoir computers to distinguish chaotic signals, Phys. Rev. E 98, 052209 (2018).

[24] K. Nakai and Y. Saiki, Machine-learning inference of fluid variables from data using reservoir computing, Phys. Rev. E 98 , 023111 (2018).

[25] R. S. Zimmermann and U. Parlitz, Observing spatio-temporal dynamics of excitable media using reservoir computing, Chaos 28, 043118 (2018).

[26] T. Weng, H. Yang, C. Gu, J. Zhang, and M. Small, Synchronization of chaotic systems and their machine-learning models, Phys. Rev. E 99, 042203 (2019).

[27] A. Griffith, A. Pomerance, and D. J. Gauthier, Forecasting chaotic systems with very low connectivity reservoir computers, Chaos 29, 123108 (2019).

[28] J. Jiang and Y.-C. Lai, Model-free prediction of spatiotemporal dynamical systems with recurrent neural networks: Role of network spectral radius, Phys. Rev. Research 1, 033056 (2019).

[29] H. Fan, J. Jiang, C. Zhang, X. Wang, and Y.-C. Lai, Long-term prediction of chaotic systems with machine learning, Phys. Rev. Research 2, 012080 (2020).

[30] C. Zhang, J. Jiang, S.-X. Qu, and Y.-C. Lai, Predicting phase and sensing phase coherence in chaotic systems with machine learning, Chaos 30, 083114 (2020).

[31] Y. Guo, H. Zhang, L. Wang, H. Fan, J. Xiao, and X. Wang, Transfer learning of chaotic systems, Chaos 31, 011104 (2021).

[32] R. Cestnik and M. Abel, Inferring the dynamics of oscillatory systems using recurrent neural networks, Chaos 29, 063128 (2019).

[33] L.-W. Kong, H.-W. Fan, C. Grebogi, and Y.-C. Lai, Machine learning prediction of critical transition and system collapse, Phys. Rev. Research 3, 013090 (2021).

[34] J. Z. Kim, Z. Lu, E. Nozari, G. J. Pappas, and D. S. Bassett, Teaching recurrent neural networks to infer global temporal structure from local examples, Nat. Mach. Intell. 3, 316 (2021).

[35] D. Patel, D. Canaday, M. Girvan, A. Pomerance, and E. Ott, Using machine learning to predict statistical properties of nonstationary dynamical processes: System climate, regime transitions, and the effect of stochasticity, Chaos 31, 033149 (2021).

[36] See Supplemental Material at http://link.aps.org/supplemental/ 10.1103/PhysRevResearch.3.023237 for the details.

[37] K. Kaneko, Theory and Application of Coupled Map Lattices, (Wiley, Chichester, 1993).

[38] E. N. Lorenz, Deterministic nonperiodic flow, J. Atmos. Sci. 20, 130 (1963).

[39] K. McCann and P. Yodzis, Nonlinear dynamics and population disappearances, Am. Nat. 144, 873 (1994). 
[40] M. G. Rosenblum, A. S. Pikovsky, and J. Kurths, Phase Synchronization of Chaotic Oscillators, Phys. Rev. Lett. 76, 1804 (1996).

[41] M. Rosenblum and A. Pikovsky, Efficient determination of synchronization domains from observations of asynchronous dynamics, Chaos 28, 106301 (2018).
[42] R.-Q. Su, X. Ni, W.-X. Wang, and Y.-C. Lai, Forecasting synchronizability of complex networks from data, Phys. Rev. E 85, 056220 (2012).

[43] W. Wang, Y.-C. Lai, and C. Grebogi, Data based identification and prediction of nonlinear and complex dynamical systems, Phys. Rep. 644, 1 (2016). 